\title{
Variation in crossing-over rates across chromosome 4 of Arabidopsis thaliana reveals the presence of meiotic recombination "hot spots"
}

\author{
Jan Drouaud, ${ }^{1}$ Christine Camilleri, ${ }^{1,2}$ Pierre-Yves Bourguignon, ${ }^{3}$ Aurélie Canaguier, ${ }^{1,6}$ \\ Aurélie Bérard, ${ }^{1,2}$ Daniel Vezon, ${ }^{1}$ Sandra Giancola, ${ }^{1,2}$ Dominique Brunel, ${ }^{1,2}$ \\ Vincent Colot, ${ }^{4}$ Bernard Prum, ${ }^{3}$ Hadi Quesneville, ${ }^{5}$ and Christine Mézard ${ }^{1,7}$ \\ ${ }^{1}$ Station de Génétique et d'Amélioration des Plantes, Institut Jean-Pierre Bourgin, Institut National de la Recherche Agronomique \\ (INRA), 78026, Versailles cedex, France; ${ }^{2}$ INRA/CNG, 91057 Evry cedex, France; ${ }^{3}$ Laboratoire Statistique et Génome, UMR 8071 \\ Centre National de la Recherche Scientifique (CNRS)-INRA-Université Evry Val d'Essonne (UEVE), 91000 Evry, France; ${ }^{4}$ Unité de \\ Recherche en Génomique Végétale (URGV), INRA/CNRS/UEVE, CP5708, 91057 Evry cedex, France; ${ }^{5}$ Laboratoire de Dynamique \\ du Génome et Evolution, Institut Jacques Monod, 75251 Paris cedex 05, France
}

\begin{abstract}
Crossover (CO) is a key process for the accurate segregation of homologous chromosomes during the first meiotic division. In most eukaryotes, meiotic recombination is not homogeneous along the chromosomes, suggesting a tight control of the location of recombination events. We genotyped 71 single nucleotide polymorphisms (SNPs) covering the entire chromosome 4 of Arabidopsis thaliana on 702 F2 plants, representing 1404 meioses and allowing the detection of $1171 \mathrm{COs}$, to study $\mathrm{CO}$ localization in a higher plant. The genetic recombination rates varied along the chromosome from $0 \mathrm{cM} / \mathrm{Mb}$ near the centromere to $20 \mathrm{cM} / \mathrm{Mb}$ on the short arm next to the NOR region, with a chromosome average of $4.6 \mathrm{cM} / \mathrm{Mb}$. Principal component analysis showed that $\mathrm{CO}$ rates negatively correlate with the $\mathrm{G}+\mathrm{C}$ content $\left(P=3 \times 10^{-4}\right)$, in contrast to that reported in other eukaryotes. $\mathrm{COs}$ also significantly correlate with the density of single repeats and the $\mathrm{CpG}$ ratio, but not with genes, pseudogenes, transposable elements, or dispersed repeats. Chromosome 4 has, on average, $1.6 \mathrm{CO}$ ser meiosis, and these COs are subjected to interference. A detailed analysis of several regions having high $\mathrm{CO}$ rates revealed "hot spots" of meiotic recombination contained in small fragments of a few kilobases. Both the intensity and the density of these hot spots explain the variation of $\mathrm{CO}$ rates along the chromosome.
\end{abstract}

[Supplemental material is available online at www.genome.org.]

Meiotic crossovers (COs) and sister chromatid cohesion provide physical links between homologous chromosomes ensuring proper chromosome segregation during the first meiotic division. In most eukaryotes, there is always at least one $\mathrm{CO}$ per pair of homologs (obligatory crossover) (Jones 1984, 1987). Cytological, genetic, and molecular studies in many organisms have demonstrated that COs are not evenly distributed along the chromosomes (Jones 1987; Carpenter 1988; Lynn et al. 2002). The tight control of the number and/or localization of COs is crucial. Mutations that reduce $\mathrm{CO}$ formation increase chromosome nondisjunction in organisms as diverse as Saccharomyces cerevisiae, Schizosaccharomyces pombe, Caenorhabditis elegans, Drosophila melanogaster (female), Arabidopsis thaliana, and the mouse (for review, see Lynn et al. 2004).

In yeast, the distribution of meiotic recombination events (COs and noncrossover gene conversions; NCOs) along chromosomes has been studied in detail by locating DNA double-strand breaks (DSBs), which initiate meiotic recombination (Baudat and Nicolas 1997; Gerton et al. 2000). These studies showed that

6Present address: URGV, INRA/CNRS, 2, rue Gaston Crémieux, CP5708, 91057 Evry cedex, France.

${ }^{7}$ Corresponding author.

E-mail mezard@versailles.inra.fr; fax (33) 130833319.

Article published online ahead of print. Article and publication date are at http://www.genome.org/cgi/doi/10.1101/gr.4319006.
DSBs tend to be clustered in chromosomal domains away from telomeres and centromeres (Gerton et al. 2000; Borde et al. 2004). In mammals, COs are also nonrandomly distributed along the chromosomes, with alternate domains having higher or lower levels of recombination (Kong et al. 2002; Nachman 2002). The $\mathrm{CO}$ rates tend to be low near the centromeres and increase toward the telomeres. In plants, the $\mathrm{CO}$ rates also vary along chromosomes (for review, see Anderson and Stack 2002). In general, centromeric regions have low $\mathrm{CO}$ rates compared to telomeric regions. However, in plants, there have been very few highresolution studies in a single chromosome.

Many sequence parameters have been linked to the variation of $\mathrm{CO}$ rates in eukaryotes. In yeast and mammals, several studies have found a correlation between a high $\mathrm{G}+\mathrm{C}$ content and a high rate of recombination in large domains (Gerton et al. 2000; Fullerton et al. 2001; Yu et al. 2001; Kong et al. 2002; Petes and Merker 2002; Jensen-Seaman et al. 2004). However, within $2-3 \mathrm{~kb}$ of the recombination initiation site no correlation between the $\mathrm{G}+\mathrm{C}$ content and the distribution of COs in both yeast and humans was found (for review, see de Massy 2003) and, second, in human, rat, and mouse, when $\mathrm{CpG}$ ratio is included in a multiple regression analysis, correlation with the $\mathrm{G}+\mathrm{C}$ content becomes negative (Kong et al. 2002; Jensen-Seaman et al. 2004). In wheat, barley, and maize, gene-rich regions are more recombinationally active than gene-poor regions (for review, see 
Schnable et al. 1998). In humans, female CO rates are not correlated with gene density on chromosome 21 (Lynn et al. 2000) whereas male CO rates are correlated, suggesting a different type of control. There are also conflicting results when correlating the density of transposable elements (TEs) and recombination rates (see Wright et al. 2003). Nevertheless, differences in meiotic CO rates between the sexes have been demonstrated in many higher eukaryotes (Lenormand and Dutheil 2005). Therefore, the primary DNA sequence itself cannot explain all of the variation of meiotic recombination.

In $S$. cerevisiae and $S$. pombe, hot spots have been defined as small DNA fragments of 1-2 kb, centered around meiotic DSBs that are repaired, using the homologous chromosome, to produce COs or NCOs (Keeney 2001). In mice, humans, and plants, several such regions have been studied in detail and have been found to share common features with hot spots described in yeast. These include high level of COs and NCOs clustered in small segments (1-2 kb) and a lack of clear consensus sequences (de Massy 2003; Kauppi et al. 2004; Rafalski and Morgante 2004). The distribution of meiotic hot spots along chromosomes is uneven, which suggests a local control of DSB formation. A lot of effort has been made recently to characterize this finescale variation of recombination rates mainly in humans but also in other eukaryotes for various reasons among which is to gain insight into the underlying mechanisms, to assist association studies, or to improve inferences from polymorphism data about selection and population history. However, except for $S$. cerevisiae, only a few regions have been characterized at the molecular level in other eukaryotes and more genomewide studies are needed to unravel the determinants of hot spot activity.

The availability of the Arabidopsis genome sequence (The Arabidopsis Genome Initiative 2000) and the recent development of powerful high-throughput genotyping techniques (Gut 2001; Kwok 2001), allow us to determine precisely the location and rates of COs on one chromosome. Here, we show that $\mathrm{CO}$ rates are highly variable on chromosome 4 of Arabidopsis, with some regions having five times more COs than the chromosome average. The $\mathrm{CO}$ rates significantly negatively correlate with the $\mathrm{G}+\mathrm{C}$ content and also significantly correlate with the density of single repeats and with CpG ratio. However, they do not correlate significantly either with genes, pseudogenes, transposable elements, or dispersed repeats. Our data also confirm that COs are subjected to interference on chromosome 4. Finally, we provide evidence of meiotic recombination hot spots and show that both their activity and density contribute to the variation of the $\mathrm{CO}$ rates.

\section{Results}

Chromosome 4 of $A$. thaliana is the smallest of its five chromosomes and presents several remarkable features (Fig. 1). It has an acrocentric architecture with a long arm $14.6 \mathrm{Mb}$ long and short arm about $8 \mathrm{Mb}$ long tipped by the nucleolar organizer region (NOR). This region is about 3.6-4 Mb long and is constituted of almost homogeneous ribosomal DNA repeats (Haberer et al. 1996). The available short arm sequence starts in the last proximal copy of the rDNA repeat (Mayer et al. 1999; The Arabidopsis Information Resource, http://www.arabidopsis.org/). In some accessions, including Columbia (Col) but not Landsberg (Ler), the short arm has a heterochomatic region, called the "knob," identified cytologically (Fransz et al. 2000), primarily comprising transposable elements, in which a few genes are insulated (Mayer et al. 1999; Lippman et al. 2004). Moreover, an approximately $1.5-\mathrm{Mb}$-long region of the short arm, including the knob, is inverted between the two accessions, Col and Ler (Fransz et al. 2000).

We genotyped a population of 736 F2 plants resulting from a cross between Col and Ler (see Methods) with 71 SNPs (Supplemental Table 1) chosen from the Monsanto database (Jander et al. 2002) to be evenly spaced on the Arabidopsis chromosome 4. The average interval between two SNPs was $204 \mathrm{~kb}$ on the long arm (60 SNPs) and $239 \mathrm{~kb}$ on the short arm (11 SNPs).

\section{Variation of $\mathrm{CO}$ rates across chromosome 4}

After SNP genotyping, we analyzed the variation in $\mathrm{CO}$ rates in 702 plants (34 plants had missing data for more than 24 markers and were thus discarded). On average, we genotyped 666 plants (thus representing 1332 meioses because in an F2 plant each chromosome comes from an independent meiosis) per interval. We verified that there was no bias in the segregation of each marker. The cumulated genetic distance of the chromosome was estimated to be $83.9 \mathrm{cM}$, of which $69 \mathrm{cM}$ corresponded to the long arm (Supplemental Table 2).

As the intervals were small, the genetic length of each interval can be simply calculated by dividing the number of recombinant chromosomes by the number of meioses analyzed. Genetic recombination varied greatly along the chromosome, from
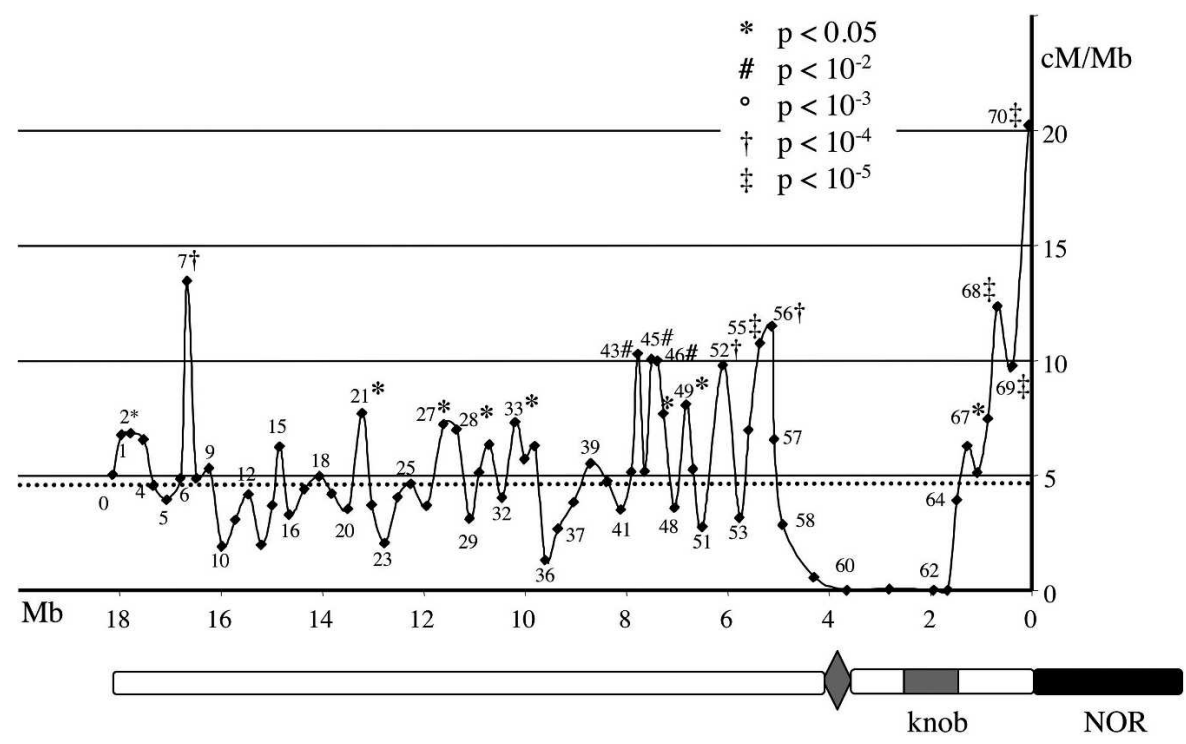

Figure 1. Variation of the $\mathrm{CO}$ rates on chromosome 4 of $A$. thaliana. The numbers refer to the intervals given in Supplemental Table 2. The dotted line represents the average CO rate on chromosome $4(4.6 \mathrm{cM} / \mathrm{Mb})$. A schematic representation of chromosome 4 of $A$. thaliana is aligned with the diagram. (Black box) NOR (nucleolar organizer region), (gray box) heterochromatic knob, (diamondshaped box) centromere. 
$0 \mathrm{cM} / \mathrm{Mb}$ next to the centromere, to $20.2 \mathrm{cM} / \mathrm{Mb}$ next to the NOR (Supplemental Table 2; Fig. 1). The frequencies of COs in different intervals could not be directly compared because of both the variation in interval length and the number of analyzed chromosomes. Therefore, we developed a statistical approach to unambiguously identify intervals that were significantly either "colder" or "hotter" than the chromosome average. The approach is based on a simply binomial model of the number of COs in each interval, so that the "temperature" of an interval is determined by the probability that the number of COs in it exceeds the expected one, under the assumption that the recombination rate is constant along the chromosome. We implemented a statistical program (TETRA) to compute both the average number of COs per nucleotide, and the significance of the observed values from the binomial model (see Methods).

TETRA calculated an average of $4.6 \times 10^{-8} \mathrm{COs} /$ nucleotide, which is, on average, $1 \mathrm{cM}$ for $217 \mathrm{~kb}$ for chromosome 4 . Among the 70 intervals tested, TETRA identified 30 intervals with a significant deviation from the average rate of COs; 12 intervals had a significantly lower rate (cold) and 18 had a significantly higher rate (hot) $(P>0.95$ and $P<0.05$ for the cold and hot intervals, respectively; Supplemental Table 2). The hot intervals were not randomly distributed: four (intervals 67-70) were clustered on the short arm next to the NOR and eight (intervals 43-56) were clustered in a $3-\mathrm{Mb}$ region on the long arm next to the centromere (Fig. 1). There was almost no genetic recombination in the centromeric and inverted region (intervals 58-63) and no clustering of the cold intervals was observed outside the centromeric region. In the middle of the long arm, there were alternate hot and cold intervals, although the "temperature" of most of these intervals was not significantly different from the chromosome average. In summary, the COs were unevenly distributed along chromosome 4 with alternating hot and mildly cold regions.

\section{Correlation of $\mathrm{CO}$ rates with primary sequence features}

We performed a principal component analysis to determine the most relevant genome features that correlated with the observed CO frequencies. The genes, pseudogenes, $\mathrm{G}+\mathrm{C}$ content, and $\mathrm{CpG}$ log ratio, as well as repeated sequences, such as transposable elements (TEs) and single repeats (SSR), were carefully listed from both publicly available data and in-house computed analysis (see Methods). In each interval, we took the $\mathrm{G}+\mathrm{C}$ content and the CpG ratio (see Methods) and calculated the density of each of the other features. We analyzed the whole chromosome, excluding the intervals 60-63 contained in the inverted region. On the first principal component axis, accounting for $46.4 \%$ of the variation, we found that gene, pseudoare indicated in blue. gene, and TE densities contribute the most to the composition diversity of the intervals (Fig. 2). However, this axis shows that these features do not occur randomly along the chromosome, but follow two opposite gradients: The gene density is low in the pericentromeric and subtelomeric regions and high in the middle of chromosome arms, whereas the opposite is true for pseudogene and TE density. On the second principal component axis, adding $22.6 \%$ to the explained variation, the GC content and the CpG ratio appear to be more relevant to $\mathrm{CO}$ rates' variation. The intervals showing a significantly higher rate of COs tend to cluster regions of low $\mathrm{G}+\mathrm{C}$ content where the $\mathrm{CpG}$ ratio is high. Conversely, the intervals with a low $\mathrm{CO}$ rate cluster in regions of high $\mathrm{G}+\mathrm{C}$ content where the $\mathrm{CpG}$ ratio is low (Fig. 2).

A regression analysis carried out between the $\mathrm{CO}$ rate and the $\mathrm{G}+\mathrm{C}$ content or CpG ratio confirmed these trends with $R^{2}$ of $0.18\left(P=3 \times 10^{-4}\right)$ for $\mathrm{G}+\mathrm{C}$ content and an $R^{2}$ of 0.20 $\left(P=1.3 \times 10^{-4}\right)$ for the $\mathrm{CpG}$ ratio. The regression was stronger when analyzing only the long arm of the chromosome, with $R^{2}=0.36\left(P=4 \times 10^{-7}\right)$ for $\mathrm{G}+\mathrm{C}$ content and $R^{2}=0.22$ $\left(P=1.7 \times 10^{-4}\right)$ for the $\mathrm{CpG}$ ratio. Of the other regressions tested (gene density, pseudogenes, etc.), only the SSR density had a significant correlation with CO rates $\left(R^{2}=0.13 ; P=3 \times 10^{-3}\right)$. Therefore, unlike the results obtained in several other eukaryotes, in which a high $\mathrm{CO}$ rate tends to correlate with a high $\mathrm{G}+\mathrm{C}$ content, we suggest that on chromosome 4 of $A$. thaliana a high

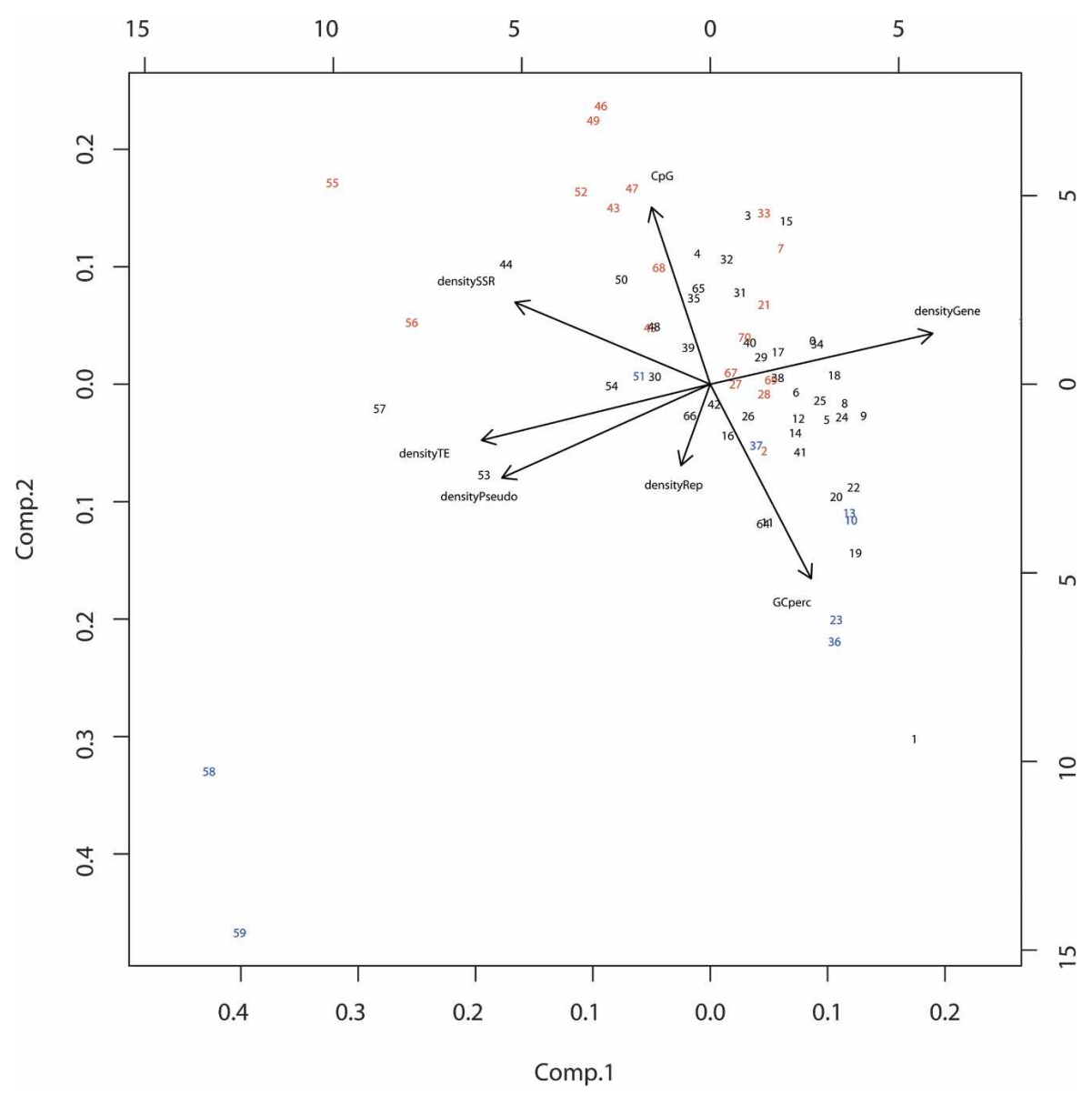

Figure 2. Principal component analysis of chromosome 4 of $A$. thaliana. Numbers refer to the intervals given in Figure 1 and Supplemental Table 2. Hot intervals are indicated in red; cold intervals

\section{Genome Research} www.genome.org 


\begin{tabular}{|c|c|c|c|c|}
\hline $\cos /$ plant & $\begin{array}{l}\text { COs/chromatid: } \\
\text { non ambiguous } \\
\text { (number of plants) }\end{array}$ & Examples & $\begin{array}{c}\text { COs/chromatidd } \\
\text { ambiguous } \\
\text { (number of plants) }\end{array}$ & Examples \\
\hline 0 & $\begin{array}{c}0+0 \\
(100)\end{array}$ & & none & \\
\hline 1 & $\begin{array}{c}0+1 \\
(223)\end{array}$ & & none & \\
\hline 2 & $\begin{array}{l}1+1 \\
(97)\end{array}$ & & & \\
\hline 2 & $\begin{array}{l}0+2 \\
(38)\end{array}$ & & (123) & \\
\hline 3 & $\begin{array}{l}1+2 \\
(57)\end{array}$ & & $\begin{array}{c}3: \\
0+3 \\
\text { or } \\
2+1 \\
(41)\end{array}$ & or \\
\hline$>3$ & none & & $\begin{array}{l}>3 \\
(23)\end{array}$ & \\
\hline
\end{tabular}

Figure 3. Number of $\mathrm{CO}$ events per chromatid deduced from the genotype of an F2 plant. When an F2 plant displays two COs, either the extremities of both chromosomes are homozygous, and the COs are on one chromatid or both, or the extremities are heterozygous and there is ambiguity between one event on each chromatid or two events on the same chromatid. A similar approach has been used to analyse F2 plants that displays three COs. When an F2 plant displays more than three COs, the recombination history cannot be inferred from the genotype. In the columns "COs/chromatid," the numbers in parentheses represent the number of plants in each category. (Light gray box) plants used in the interference analysis. (Dark gray box) plants containing a chromatid with two COs but that could not be used in the interference analysis.

CO rate correlates with a low $\mathrm{G}+\mathrm{C}$ content. The $\mathrm{CpG}$ ratio and SSR density also weakly correlate with CO rates.

\section{Interference on chromosome 4}

We obtained 1171 COs for 1404 analyzed meioses. This corresponded to an average of 0.8 events per chromatid and per meiosis, corresponding to 1.6 COs per pair of homologous chromosomes (bivalents) per meiosis. There were, on average, 1.3 events on the long arm and 0.3 events on the short arm. However, if we take into account the $1.5 \mathrm{Mb}$ that are inverted between the two parental lines, and therefore "forbidden" from forming and/or recovering COs, the ratio of COs per megabase on the short arm was double that of the long arm (0.18 vs. 0.09).

For 515 pairs of chromosomes, we were able to determine unequivocally the number of exchanges that each chromatid had undergone (0, 1, or 2 COs) during meiosis (Fig. 3). For 123 pairs of chromosomes harboring two exchanges, we could not unambiguously attribute the recombination events to one or the other chromatid. We reassigned them either to the " $1+1$ " or the " $2+0$ " class (see Fig. 3 ) on the basis of the prorata between the sizes of these latter classes in the nonambiguous class with two exchanges. The 41 pairs that exhibit three exchanges that could not be credited to one or the other chromatid were considered to fall in the " $2+1$ " class (that is, we assumed " $3+0$ " pairs to be very rare). For the remaining pairs (23), which display four or more $\mathrm{CO}$, we could not attribute $\mathrm{CO}$ unambiguously to parental chromosomes, so we discarded them. As expected, one exchange event was the most common occurrence (692 chromatids). Furthermore, we compared the observed distribution of the number of COs to what is expected under a Poisson distribution (Supplemental Table 3), Test of $\chi^{2}$ goodness-of-fit shows that the null Poisson hypothesis can be strongly rejected $\left(\chi^{2}=121.8\right.$, $\left.P<5 \times 10^{-4}\right)$. Hence, we can conclude that multiple COs do not occur on chromosome 4 independently one from each other.

For each of the 38 plants having two precisely located COs on the same chromatid (Fig. 3, light gray box), we calculated the genetic distance between the two COs. The distance varied from 1.17 to $62.8 \mathrm{cM}$ with a mean distance of $44.1 \mathrm{cM}$. The mean expected value for randomly distributed double COs was onethird of the chromosome, being $27.9 \mathrm{cM}$ (see Methods). We then classified the 38 plants into four groups: group 1, with events separated by less than $25 \%$ of the chromosome $(0-21 \mathrm{cM})$; group 2 , with events separated by more than $25 \%$ but less than $50 \%$ of the chromosome (21-42 cM); group 3, with events separated by more than $50 \%$ but less than $75 \%$ of the chromosome (42-63 $\mathrm{cM}$ ); and group 4, with events separated by more than $75 \%$ of the chromosome (63-83.9 cM) (Fig. 4). We compared the observed distribution with the expected distribution if COs were located independently of each other. We found a very strong probability $\left(\chi^{2}=27.9, P<5 \times 10^{-3}\right)$ that double COs were not located independently of each other. The same analysis on only the long arm also showed that the observed distribution and the observed mean distance $(36 \mathrm{cM})$ were very different from the theoretical values (23 cM; data not shown). We then looked at the effect of the centromere on interference. For the 12 chromosomes having one $\mathrm{CO}$ on the short arm and the other on the long arm, the mean distance was $59.6 \mathrm{cM}$, that is, $70 \%$ of the genetic length of the chromosome, while the mean distance between two COs occurring on the long arm represents $52 \%$ of the genetic length of the long arm. These results confirm that CO location on chromosome 4 is affected by interference and that the centromere is not a barrier to interference.

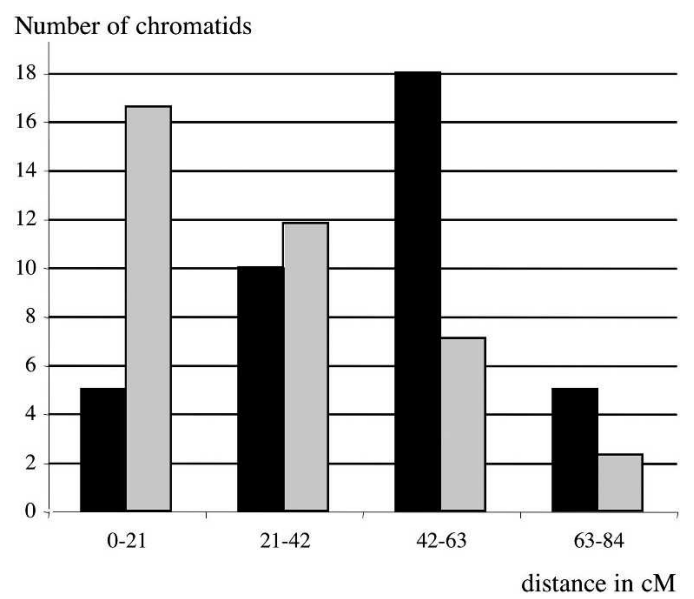

Figure 4. Distribution of the distances in centiMorgans between double COs. (Histogram in black) observed distribution of double COs in our F2 (see text); (histogram in gray) theoretical distribution of double $\mathrm{COs}$ if the position of one $\mathrm{CO}$ is independent of the second (Methods). 


\section{Evidence for the existence of hot spots of recombination}

We further investigated several of the 14 intervals having the highest $\mathrm{CO}$ rates together with one interval with a slightly above average $\mathrm{CO}$ rate and one cold interval. For each interval, we genotyped the corresponding recombinant plants using a set of SNP or indel markers, giving precise locations of the exchange points. We divided the hottest interval (interval 70; Fig. 1) into 15 parts to map the COs at a precision of a few kilobases (Fig. 5A). We found a clearly nonhomogeneous distribution of exchange events. Two very small fragments (3.4 and $3.2 \mathrm{~kb}) 20 \mathrm{~kb}$ apart exhibited a very high rate of COs $(>85 \mathrm{cM} / \mathrm{Mb})$, being 15 times higher than the chromosome average $(4.6 \mathrm{cM} / \mathrm{Mb})$ and four times higher than the interval average $(20.2 \mathrm{cM} / \mathrm{Mb})$. We found that two other fragments in interval 70 had moderately high rates of genetic recombination $(40$ and $55 \mathrm{cM} / \mathrm{Mb}, 8$ to 10 times the chromosome average). We also analyzed another hot interval (interval 21, Fig. 5B) in the middle of the long arm (Fig. 1). We found one DNA fragment displaying a large increase of genetic recombination in this interval. The recombination rates in the remainder of this interval were mostly lower than the chromosome average. We also observed the same type of "spotty" CO distribution in the other hot intervals that we investigated $(7,55$, 56 and 68, 69; data not shown).

We then analyzed interval 57 (Fig. 1), which did not appear to have a significantly high rate of genetic recombination when analyzed by TETRA $(6.6 \mathrm{cM} / \mathrm{Mb} ; P=0.08)$. We found one DNA fragment of $12 \mathrm{~kb}$ displaying a high rate of genetic recombination $(40 \mathrm{cM} / \mathrm{Mb})$ whereas the remainder of the interval displayed $\mathrm{CO}$ rates below the chromosome average (Fig. 5C). Interval 37 was found to be significantly cold when analyzed by TETRA (2.7 $\mathrm{cM} / \mathrm{Mb} ; P>0.98)$. We performed the same kind of analysis as for
A

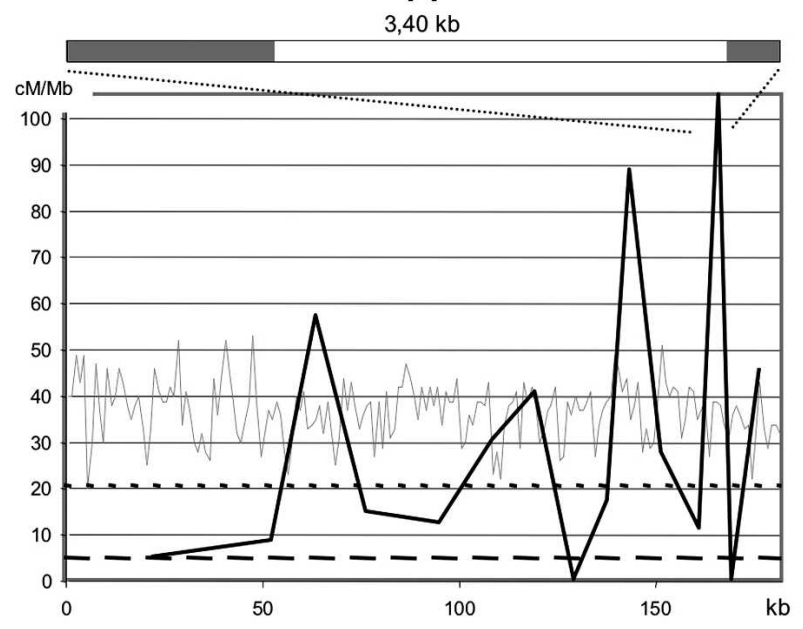

B

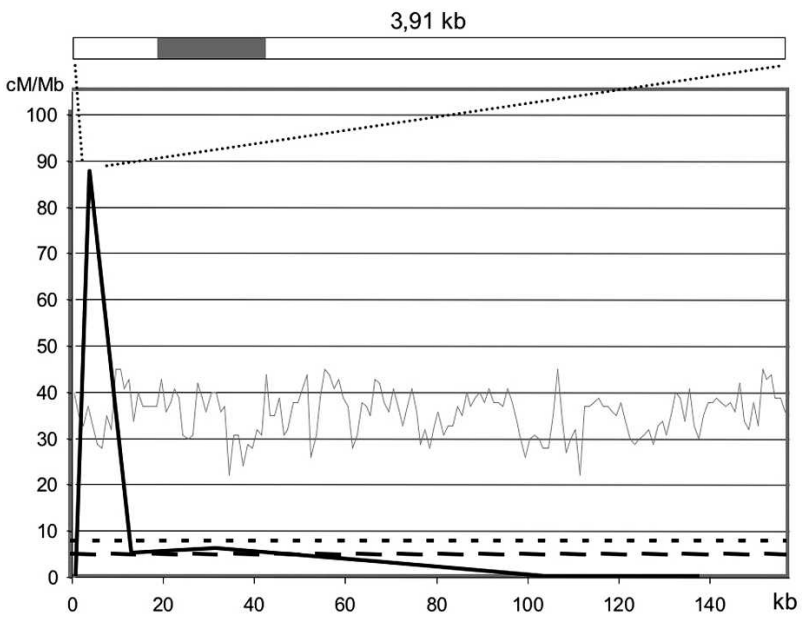

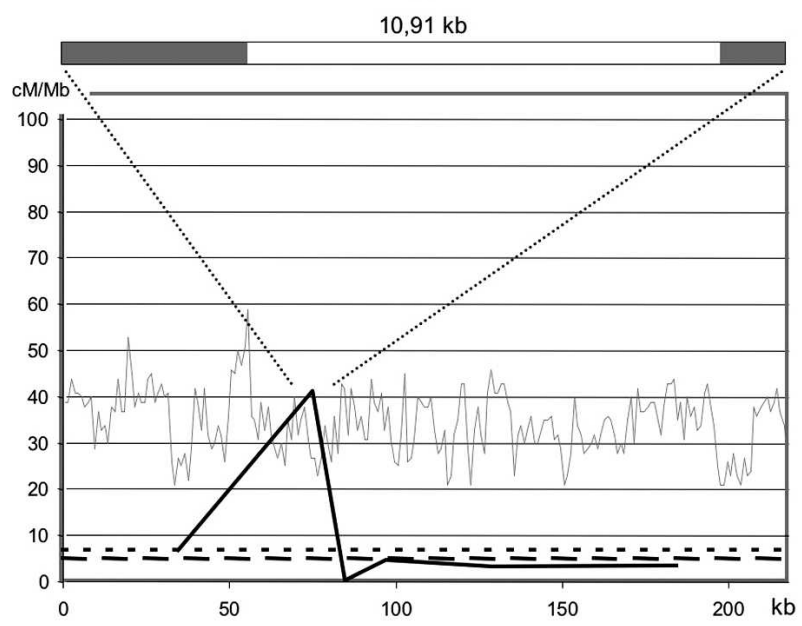

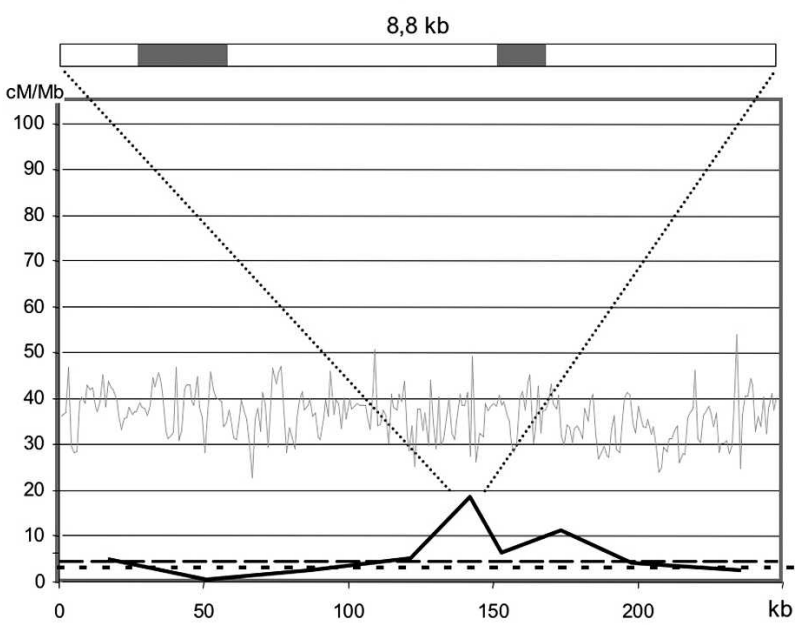

Figure 5. Fine-scale analysis of the distribution of CO breakpoints in four intervals. $(A)$ interval $70: 44.5 \mathrm{~kb}-3 ; 16.4 \mathrm{~kb}-2 ; 6.2 \mathrm{~kb}-5 ; 19.5 \mathrm{~kb}-4 ; 17.5 \mathrm{~kb}-3$; 9.4kb-4; $12.3 \mathrm{~kb}-7 ; 8.1 \mathrm{~kb}-0 ; 8.3 \mathrm{~kb}-2 ; 3.2 \mathrm{~kb}-4 ; 13.0 \mathrm{~kb}-5 ; 6.4 \mathrm{~kb}-1 ; 3.4 \mathrm{~kb}-5 ; 3.3 \mathrm{~kb}-0 ; 11.0 \mathrm{~kb}-7$. (B) interval $21: 2.5 \mathrm{~kb}-0 ; 3.9 \mathrm{~kb}-5 ; 13.8 \mathrm{~kb}-1 ; 23.6 \mathrm{~kb}-2 ;$ 45.7kb-2; 29.2kb-0; 38.6kb-0. (C) interval 57: 69.5kb-6; 12.2kb-7; 7.1kb-0; 17.4kb-1; 46.5kb-2; 65.0kb-3. (D) interval 37: 35.2kb-2; 32.7kb-0; $38.1 \mathrm{~kb}-1 ; 8.8 \mathrm{~kb}-2 ; 12.6 \mathrm{~kb}-1 ; 28.3 \mathrm{~kb}-1 ; 20.2 \mathrm{~kb}-1 ; 49.2 \mathrm{~kb}-2 ; 38.7 \mathrm{~kb}-1$. For each interval the size of each DNA fragment is given and the number of recombinant plants. (Light gray line) $\mathrm{G}+\mathrm{C}$ content calculated in 1-kb windows. (Black line) $\mathrm{CO}$ rates in centiMorgans per megabase. (Small dotted line) interval COs rate average. (Large dotted line) chromosome $4 \mathrm{COs}$ rate average. Above each major peak is the gene organization of the fragment. (Gray box) gene.

\section{Genome Research} www.genome.org 
the other intervals. We found a dispatch of the $12 \mathrm{CO}$ exchanges in 9 of the 10 fragments studied (Fig. 5D) with a maximum of two events in an 8.8-kb fragment. This small fragment seems to exhibit a slightly higher $\mathrm{CO}$ rate than the genome average (Fig. 5D). However, more plants would be needed to confirm this difference. For the four regions analyzed, hot spots did not seem to correlate with $\mathrm{G}+\mathrm{C}$ content or gene organization (Fig. 5A-D).

\section{Discussion}

We obtained a very detailed genetic map of chromosome 4 of $A$. thaliana by genotyping a series of 71 SNP markers on 702 F2 plants issued from an F1 Col/Ler hybrid. The total size of the genetic map was estimated at $83.9 \mathrm{cM}$, which is consistent with other maps obtained from crosses of the same accessions: the classical map (76 cM; Meinke et al. 1998), the RFLP map (74.4 cM; Schmidt et al. 1995; Liu et al. 1996), tetrad analysis using the quartet mutation (85 cM; Copenhaver et al. 1998; Lam et al. 2005), and first versions of the RIL genetic map ( 76 cM; Lister and Dean 1993).

We found that, on average, a chromosome 4 bivalent undergoes 1.6 crossovers per meiosis. Copenhaver et al. found an average of $1.5 \mathrm{COs}$ on chromosome 4 in male meiosis in a Col/ Ler cross (Copenhaver et al. 1998; Lam et al. 2005). Meiotic recombination has also been assessed using cytology by recording the numbers and locations of chiasmata on metaphase I bivalents in pollen mother cells of several accessions, including Col and Ler (Sanchez-Moran et al. 2002). Both the genetic and cytological methods gave consistent results, with the mean chiasma frequency being 1.6 for chromosome 4 . Therefore, $\mathrm{CO}$ frequency on chromosome 4 during meiosis of a Col/Ler F1 hybrid is not greatly different from that in the parents.

In most eukaryotes, "positive interference" (i.e., the probability of COs occurring next to each other is lower than expected) affects the distribution of multiple COs on a single chromosome (see Zickler and Kleckner 1999). However, not all the COs seem to interfere, and recent data suggest two pathways for crossovers in $S$. cerevisiae, in humans, and in A. thaliana: one pathway being sensitive to interference (class I) and the other insensitive (class II) (Copenhaver et al. 2002; Housworth and Stahl 2003; Higgins et al. 2004; Hollingsworth and Brill 2004; Stahl et al. 2004; Lam et al. 2005; Mercier et al. 2005). We show also that COs are subjected to interference on chromosome 4 . Double COs on the same chromatid are significantly further than one-third of the chromosome length apart, contrary to what is expected for randomly distributed COs. In addition, our results suggest that interference is insensitive to centromere as previously proposed by Colombo and Jones (1997) and that the centromere may increase the strength of interference on chromosome 4. However, there is not complete interference, as we observed double COs only a few centiMorgans apart. We could assume, as suggested by previous studies (Copenhaver et al. 2002; Lam et al. 2005) that these close double COs are insensitive to interference and the distant double COs are sensitive to interference. In yeast, the level of interference has been shown to depend on the size of the chromosome with the short chromosomes harboring less interference (Kabback et al. 1999). However, the disparity in size of the chromosomes is less pronounced in Arabidopsis, with the shortest chromosome being more than twothirds of the size of the longest chromosome, while in yeast there is a fourfold difference in size. Moreover, Lam et al. (2005) recently provided evidences that in Arabidopsis NOR-bearing chro- mosomes (i.e., chromosomes 2 and 4) exhibit more interference than the others and suggested that the NOR region itself rather than the size of the chromosome could influence interference. Further analyses are needed to determine whether interference varies along the chromosome, as recently suggested in a study on rice (Esch 2005).

Numerous studies have attempted to understand the factors responsible for genetic recombination variations and to identify primary sequence features that may correlate with this variability. In many sexual organisms, such as mammals, birds, yeast, drosophila, and nematodes, positive correlations between the $\mathrm{CO}$ rates and $\mathrm{G}+\mathrm{C}$ content have been observed at the scale of several hundred of kilobases (Hurst et al. 1999; Gerton et al. 2000; Fullerton et al. 2001; Marais et al. 2001; Takano-Shimizu 2001; Yu et al. 2001; Birdsell 2002; Kong et al. 2002; JensenSeaman et al. 2004). Gerton et al. (2000) suggested that regions of high $\mathrm{G}+\mathrm{C}$ content stimulate recombination. Alternatively, several recent studies have proposed that high levels of recombination may create regions with high $\mathrm{G}+\mathrm{C}$ content, probably through a biased gene conversion (BGC) toward $\mathrm{G}+\mathrm{C}$. In other words, meiotic recombination modifies the base composition through the average density of recombination hot spots (see below; Galtier et al. 2001; Birdsell 2002; Montoya-Burgos et al. 2003; Meunier and Duret 2004). However, in humans, rats, and mice, when CpG ratio is included in a multiple regression model, the correlation with the $\mathrm{G}+\mathrm{C}$ content becomes negative (Kong et al. 2002; Jensen-Seaman et al. 2004).

In contrast, we found that regions of low $\mathrm{G}+\mathrm{C}$ content and high CpG ratio on chromosome 4 of $A$. thaliana tend to have higher rates of genetic recombination. Therefore, the BGC hypothesis suggested to explain the correlation found in other eukaryotes may not apply in Arabidopis. However, homologs of genes believed to participate in $\mathrm{G}+\mathrm{C}$-biased mismatch repair in other organisms exist in the genome of Arabidopsis (Birdsell 2002). It is also possible that in Arabidopsis BGC cannot affect the nucleotide content due to the high level of inbreeding of the plant that does not favor the formation of heteroduplex DNAs. However, this would explain an absence of correlation but not a negative correlation. In contrast to our results, a study recently reported no correlation between the $\mathrm{G}+\mathrm{C}$ content and $\mathrm{CO}$ rates in Arabidopsis (Marais et al. 2004). We suggest that our observation is due to the higher precision of our recombination map because we studied 702 plants compared to the 101 RILs used in the study of Marais et al. (2004). Therefore, our study in Arabidopsis questions the assumptions made for $\mathrm{G}+\mathrm{C}$ correlation and so, the problem of causation remains an open query. Data from more species are needed and may reveal a species-specific lineage in the evolution of recombination.

A fine-scale analysis of several intervals showed peaks in crossover activity. For example, in the hottest interval (interval 70; Fig. 1), CO breakpoints are found in 12 of the 14 fragments tested, even though there is clustering in two small regions $20 \mathrm{~kb}$ apart (Fig. 5A). In other intervals, including one not having a significantly high CO rate, one small DNA fragment accounts for most of the genetic recombination of the interval. In the genome of $A$. thaliana, this punctuate distribution of $\mathrm{CO}$ activity strongly suggests recombination hot spots where recombination events group around an initiation site. In plants, several hot spots of $\mathrm{CO}$ activity have been described. The $140-\mathrm{kb}$ a1-sh 2 region in maize has peaks of $\mathrm{CO}$ activity (three to six times the genome average) in three small intervals (1.7-3.4 kb) (Yao et al. 2002). Other loci in maize or in rice, such as bronze or waxy, show some properties 
of hot spots (Dooner and Martinez-Ferez 1997; Okagaki and Weil 1997; Inukai et al. 2000). For the bronze locus, unlike for yeast and mammals, it has been suggested that recombination is initiated uniformly along the gene and not at a preferential site. Therefore, although existence of hot spots seems to be the rule rather than the exception in plants and other higher eukaryotes, there may be some differences. However, all studies on higher eukaryotes looked at no more than one or two intervals that were often selected for a phenotype associated with the recombination event. Therefore, it is difficult to determine whether the observed hot spot patterns in these intervals can be applied to the whole genome. Here, we show that a punctuate distribution of hot spots is a general feature of the chromosome that is not restricted to significantly recombinogenic regions. Our results also strongly suggest that recombination is initiated at preferential sites all along the chromosome. However, both the intensity and the density of the recombination sites influence the variation of recombination, as hot regions contain one or several very hot spots whereas a mildly warm interval would contain only one mild hot spot. Cold regions may contain few spots with a higher rate of recombination than the genome average, but it remains to be demonstrated. A similar result has recently been obtained in the human genome, where strong hot spots have been detected in narrow regions of strong LD and weak hot spots in regions of strong marker association (Jeffreys et al. 2005). We have identified more than 10 small DNA fragments that may behave as hot spots on chromosome 4 of $A$. thaliana. Further experiments are needed to confirm the strength and the precise location of the initiation site of these hot spots. Their fine characterization and analysis in other genetic backgrounds is needed to determine the factors that govern their activity and distribution.

\section{Methods}

\section{F2 recombinant population construction, genomic DNA extraction}

The two Arabidopsis accessions, Columbia and Landsberg erecta, were crossed to obtain an F1 hybrid. Self-fertilization from a single F1 was carried out to obtain F2 seeds. Seeds were grown in soil in long-day conditions in the greenhouse. At the rosette stage, the whole material of $736 \mathrm{~F} 2$ plants together with plant material from the two parental accessions was collected. DNA was extracted as described (Loudet et al. 2002).

\section{Selection of SNPs}

Most of the SNPs were chosen from the Monsanto database (Jander et al. 2002). When convenient SNPs were not found in the database, DNA fragments were amplified at the desired position on the genomic DNA of the two parental accessions and sequenced to identify a SNP suitable for genotyping. A list of the SNPs used in this study is given in Supplemental Table 1. A couple of primers were designed for each SNP to obtain a PCR fragment containing the predicted SNP. A list of the PCR primers used in this study is given in Supplemental Table 1. The PCRs were carried out on the parental accession DNAs using standard conditions: $94^{\circ} \mathrm{C} 4 \mathrm{~min},\left(94^{\circ} \mathrm{C} 45 \mathrm{sec}, 52^{\circ} \mathrm{C} 45\right.$ sec, $\left.72^{\circ} \mathrm{C} 1 \mathrm{~min}\right) \times 35$ cycles, $72^{\circ} \mathrm{C}$, with Eurobio $1 \times$ reaction buffer and Taq polymerase. PCR fragments were sequenced (Genome Express) to check the presence and position of the SNPs.

\section{SNP genotyping}

At each of the SNP sites, DNA extracted from the F2 plants was genotyped either by matrix-assisted laser desorption/ionization time-of-flight (MALDI-TOF) mass spectrometry, as described by Sauer et al. (2000) or by fluorescence based techniques: the Amplifluor technology (Serological Corporation) or the TaqMan technology (Applied Biosystems). For a number of SNPs, the results obtained with one technique (usually mass spectrometry) were confirmed with one of the two other methods. The techniques used for each SNP are given in Supplemental Table 1.

\section{Statistical analysis of $\mathrm{CO}$ rates: TETRA}

We define $P$ as the probability of having a $\mathrm{CO}$ in a specific position of the chromosome, and assume that $P \ll 1$. The probability $P_{i}$ of observing one $\mathrm{CO}$ in the ith interval on a chromosome is approximated as $P L_{i}$, where $L_{i}$ stands for the length of the $i$ th interval. If the number of informative chromosomes for the interval $i$ (i.e., the number of chromosomes for which both SNPs delimiting the interval are available) is written as $V_{i}$, then the number, $N_{i}$, of COs in the ith interval is distributed according to a binomial $B\left(V_{i}, P L_{i}\right)$ under the null hypothesis that the COs rate is constant along the chromosome. More explicitly, we have for all

$$
k: P\left(N_{i}=k\right)=\left(\begin{array}{l}
V i \\
k
\end{array}\right)\left(P \times L_{i}\right)^{k}\left(1-P \times L_{i}\right)^{V i-k} .
$$

According to the observed values, $n_{i}$, of the number of COs in the different intervals, TETRA computes the average CO rate, $P$, along the whole chromosome. It then computes the $P$-value, $T_{i}$, of the observed number of COs under the above binomial model, that is:

$$
P\left(N_{i} \geq n_{i}\right)=\sum_{k=n i}^{V i}\left(\begin{array}{l}
V i \\
k
\end{array}\right)\left(P \times L_{i}\right)^{k}\left(1-P \times L_{i}\right)^{V i-k} .
$$

This $P$-value can be interpreted as the probability that the number of $\mathrm{COs}$ in the ith interval exceeds its observed value under the model of homogeneous $\mathrm{CO}$ rate along the chromosome.

\section{Statistical analysis of COs interference}

We derive the probability distribution function of the distribution of distances between the two COs by assuming that the locations of the two COs are independently uniformly distributed random variables. $L$ is the length of the chromosome, and $x$ and $y$ are locations of the two COs. $x$ and $y$ are uniformly distributed in $[0, L]$. The distance $r=(x-y)$. The distribution of $x$ and $y$ is symmetric under the exchange of $x$ and $y$; we can condition on $x>y$. The probability distribution function $P(r)$ is proportional to the length of the segment, in the $x, y$ plane, between the points of coordinates $(r, 0)$ and $(L, L-r)$, which is itself proportional to $L-r$. Imposing the normalization of the probability distribution function, we obtain $P(r)=2(1-r / L)$. The expectation value of $r$ is thus $\int_{0}^{L} P(r) r d r=L / 3$. We can deduce the probabilities $P_{1}, P_{2}, P_{3}, P_{4}$ of $r$ being in each of the four bins $[0, L / 4],[L / 4, L / 2],[L / 2,3 L / 4],[3 L / 4, L]$. For instance $P_{1}=2 \int_{0}^{1 / 4}(1-x) d x=7 / 16$. Similarly, we find $P_{2}=5 / 16, P_{3}=3 / 16$, $P_{4}=1 / 16$. These probabilities are used in the analysis of Figure 4 .

\section{Correlation studies}

The $A$. thaliana genomic sequence and its annotation were downloaded from the TIGR Web site (http://ftp.tigr.org/pub/data/ a_thaliana/ath1/). Gene and pseudogene annotations have been 
extracted from TIGR-XML files from release 5 of the genome annotation. Transposable elements have been re-annotated using the RMBLR procedure from the TE annotation pipeline described by Quesneville et al. (2005). The TE reference set used is derived from the $A$. thaliana RepeatMasker repeat library (March 6,2004 ). The same TE family consecutive TE fragments (on both the genome and the reference TE) have been automatically joined if separated by a sequence composed of more than $80 \%$ of other TE insertions (in this case we have a nested TE). Otherwise they are joined if a gap of 5000 nucleotides or a region of mismatches 500 nucleotides long separate them.

Single repeats (SSR) were found using the Tandem Repeat Finder program (Benson 1999), and repeats by a BLASTN all-byall using BLASTER and GROUPER (Quesneville et al. 2005) without using any simple link clustering coverage constraint. $\mathrm{G}+\mathrm{C}$ content and $\mathrm{CpG}$ were counted with in-house python scripts. CpG ratios were computed by taking the $\log _{10}$ of the $\mathrm{C}+\mathrm{G}$ dinucleotide frequency divided by the product of $G$ and $C$ frequencies. All data and analysis results were stored in a MySQL database and retrieved by SQL queries.

Statistical analyses were carried out using the $\mathrm{R}$ software environment (http://cran.r-project.org)

\section{Detection of hot spots}

We halved each interval by choosing a convenient SNP (or indel) either from the Monsanto database or by DNA sequencing (see above). We then sequenced the DNA fragment containing the SNP in the recombinant plants and finally distributed the plants according to their genotype within one or the other half interval. This was iteratively repeated until the location of $\mathrm{CO}$ breakpoints was obtained within a few kilobases. A list of the SNPs, indels, and corresponding primers is given in Supplemental Table 1. Genomic DNA from plants was amplified by PCR in standard conditions (see above) with an annealing temperature adapted for each set of primers.

\section{Acknowledgments}

We thank Mathilde Grelon, Raphaël Mercier, Eric Jenczewski, and Valérie Borde for critical reading of the manuscript and Sylvie Jolivet for technical assistance. All the members of the "Méiose et Recombinaison" group provided helpful comments and participated in stimulating discussions. Marc Mézard kindly provided the statistical analysis of interference. This work was supported by grants from the Institut National de la Recherche Agronomique (to C.M.) and the European Union (Epigenome Network of Excellence to V.C.).

\section{References}

Anderson, L.K. and Stack, S.M. 2002. Meiotic recombination in plants. Curr. Genomics 3: 507-525.

The Arabidopsis Genome Initiative. 2000. Analysis of the genome sequence of the flowering plant Arabidopsis thaliana. Nature 408: 796-815.

Baudat, F. and Nicolas, A. 1997. Clustering of meiotic double-strand breaks on yeast chromosome III. Proc. Natl. Acad. Sci. 94: 5213-5218.

Benson, G. 1999. Tandem repeats finder: A program to analyze DNA sequences. Nucleic Acids Res. 27: 573-580.

Birdsell, J.A. 2002. Integrating genomics, bioinformatics, and classical genetics to study the effects of recombination on genome evolution. Mol. Biol. Evol. 19: 1181-1197.

Borde, V., Lin, W., Novikov, E., Petrini, J.H., Lichten, M., and Nicolas, A. 2004. Association of Mre11p with double-strand break sites during yeast meiosis. Mol. Cell 13: 389-401.

Carpenter, A.T.C. 1988. Thoughts on recombination nodules, meotic recombination, and chiasmata. In Genetic recombination (eds. E.R.
Kucherlapati and G.R. Smith), pp. 529-548. American Society for Microbiology, Washington, DC.

Colombo, P.C. and Jones, G.H. 1997. Chiasma interference is blind to centromeres. Heredity 79: 214-227.

Copenhaver, G.P., Browne, W.E., and Preuss, D. 1998. Assaying genome-wide recombination and centromere functions with Arabidopsis tetrads. Proc. Natl. Acad. Sci. 95: 247-252.

Copenhaver, G.P., Housworth, E.A., and Stahl, F.W. 2002. Crossover interference in Arabidopsis. Genetics 160: 1631-1639.

de Massy, B. 2003. Distribution of meiotic recombination sites. Trends Genet. 19: 514-522.

Dooner, H.K. and Martinez-Ferez, I.M. 1997. Recombination occurs uniformly within the bronze gene, a meiotic recombination hotspot in the maize genome. Plant Cell 9: 1633-1646.

Esch, E. 2005. Estimation of gametic frequencies from F2 populations using the EM algorithm and its application in the analysis of crossover interference in rice. Theor. Appl. Genet. 111: 100-109.

Fransz, P.F., Armstrong, S., de Jong, J.H., Parnell, L.D., van Drunen, C., Dean, C., Zabel, P., Bisseling, T., and Jones, G.H. 2000. Integrated cytogenetic map of chromosome arm $4 \mathrm{~S}$ of $A$. thaliana: Structural organization of heterochromatic knob and centromere region. Cell 100: $367-376$

Fullerton, S.M., Carvalho, A.B., and Clark, A.G. 2001. Local rates of recombination are positively correlated with GC content in the human genome. Mol. Biol. Evol. 18: 1139-1142.

Galtier, N., Piganeau, G., Mouchiroud, D., and Duret, L. 2001. GC-content evolution in mammalian genomes: The biased gene conversion hypothesis. Genetics 159: 907-911.

Gerton, J.L., DeRisi, J., Shroff, R., Lichten, M., Brown, P.O., and Petes, T.D. 2000. Global mapping of meiotic recombination hotspots and coldspots in the yeast Saccharomyces cerevisiae. Proc. Natl. Acad. Sci. 97: 11383-11390.

Gut, I.G. 2001. Automation in genotyping of single nucleotide polymorphisms. Hum. Mutat. 17: 475-492.

Haberer, G., Fischer, T.C., and Torres-Ruiz. R.A. 1996. Mapping of the nucleolus organizer region on chromosome 4 in Arabidopsis thaliana. Mol. Gen. Genet. 250: $123-128$.

Higgins, J.D., Armstrong, S.J., Franklin, F.C., and Jones, G.H. 2004. The Arabidopsis MutS homolog AtMSH4 functions at an early step in recombination: Evidence for two classes of recombination in Arabidopsis. Genes \& Dev. 18: 2557-2570.

Hollingsworth, N.M. and Brill, S.J. 2004. The Mus81 solution to resolution: Generating meiotic crossovers without Holliday junctions. Genes \& Dev. 18: 117-125.

Housworth, E.A. and Stahl, F.W. 2003. Crossover interference in humans. Am. J. Hum. Genet. 73: 188-197.

Hurst, L.D., Brunton, C.F., and Smith, N.G. 1999. Small introns tend to occur in GC-rich regions in some but not all vertebrates. Trends Genet. 15: 437-439.

Inukai, T., Sako, A., Hirano, H.Y., and Sano, Y. 2000. Analysis of intragenic recombination at $\mathrm{wx}$ in rice: Correlation between the molecular and genetic maps within the locus. Genome 43: 589-596.

Jander, G., Norris, S.R., Rounsley, S.D., Bush, D.F., Levin, I.M., and Last, R.L. 2002. Arabidopsis map-based cloning in the post-genome era. Plant Physiol. 129: 440-450.

Jeffreys, A.J., Neumann, R., Panayi, M., Myers, S., and Donnelly, P. 2005. Human recombination hot spots hidden in regions of strong marker association. Nat. Genet. 37: 601-606.

Jensen-Seaman, M.I., Furey, T.S., Payseur, B.A., Lu, Y., Roskin, K.M., Chen, C.F., Thomas, M.A., Haussler, D., and Jacob, H.J. 2004. Comparative recombination rates in the rat, mouse, and human genomes. Genome Res. 14: 528-538.

Jones, G.H. 1984. The control of chiasma distribution. Symp. Soc. Exp. Biol. 38: 293-320.

Jones, G.H. 1987. Chiasmata. In Meiosis (ed. P.B. Moens), pp. 213-244. Academic Press, London.

Kaback, D.B., Barber, D., Mahon, J., Lamb, J., and You, J. 1999. Chromosome size-dependent control of meiotic reciprocal recombination in Saccharomyces cerevisiae: The role of crossover interference. Genetics 152: 1475-1486.

Kauppi, L., Jeffreys, A.J., and Keeney, S. 2004. Where the crossovers are: Recombination distributions in mammals. Nat. Rev. Genet. 5: $413-424$.

Keeney, S. 2001. Mechanism and control of meiotic recombination initiation. Curr. Top. Dev. Biol. 52: 1-53.

Kong, A., Gudbjartsson, D.F., Sainz, J., Jonsdottir, G.M., Gudjonsson, S.A., Richardsson, B., Sigurdardottir, S., Barnard, J., Hallbeck, B., Masson, G., et al. 2002. A high-resolution recombination map of the human genome. Nat. Genet. 31: 241-247.

Kwok, P.Y. 2001. Methods for genotyping single nucleotide polymorphisms. Annu. Rev. Genomics Hum. Genet. 2: 235-258. 
Lam, S., Horn, S.R., Radford, S.J., Housworth, E.A., Stahl, F.W., and Copenhaver, G.P. 2005. Crossover interference on NOR-bearing chromosomes in Arabidopsis. Genetics 170: 807-812.

Lenormand, T. and Dutheil, J. 2005. Recombination difference between sexes: A role for haploid selection. PLoS Biol. 3: e63.

Lippman, Z., Gendrel, A.V., Black, M., Vaughn, M.W., Dedhia, N., McCombie, W.R., Lavine, K., Mittal, V., May, B., Kasschau, K.D., et al. 2004. Role of transposable elements in heterochromatin and epigenetic control. Nature 430: 471-476.

Lister, C. and Dean, C. 1993. Recombinant inbred lines for mapping RFLP and phenotypic markers in Arabidopsis thaliana. Plant J. 4: 745-750.

Liu, Y.G., Mitsukawa, N., Lister, C., Dean, C., and Whittier, R.F. 1996. Isolation and mapping of a new set of 129 RFLP markers in Arabidopsis thaliana using recombinant inbred lines. Plant J. 10: $733-736$.

Loudet, O., Chaillou, S., Camilleri, C., Bouchez, D., and Daniel-Vedele, F. 2002. Bay-0 x Shahdara recombinant inbred line population: A powerful tool for the genetic dissection of complex traits in Arabidopsis. Theor. Appl. Genet. 104: 1173-1184.

Lynn, A., Kashuk, C., Petersen, M.B., Bailey, J.A., Cox, D.R. Antonarakis, S.E., and Chakravarti, A. 2000. Patterns of meiotic recombination on the long arm of human chromosome 21. Genome Res 10: 1319-1332.

Lynn, A., Koehler, K.E., Judis, L., Chan, E.R., Cherry, J.P., Schwartz, S., Seftel, A., Hunt, P.A., and Hassold, T.J. 2002. Covariation of synaptonemal complex length and mammalian meiotic exchange rates. Science 296: 2222-2225.

Lynn, A., Ashley, T., and Hassold, T. 2004. Variation in human meiotic recombination. Annu. Rev. Genomics Hum. Genet. 5: 317-349.

Marais, G., Mouchiroud, D., and Duret, L. 2001. Does recombination improve selection on codon usage? Lessons from nematode and fly complete genomes. Proc. Natl. Acad. Sci. 98: 5688-5692.

Marais, G., Charlesworth, B., and Wright, S.I. 2004. Recombination and base composition: The case of the highly self-fertilizing plant Arabidopsis thaliana. Genome Biol. 5: R45.

Mayer, K. Schuller, C., Wambutt, R., Murphy, G., Volckaert, G., Pohl, T., Dusterhoft, A., Stiekema, W., Entian, K.D., Terryn, N., et al. 1999. Sequence and analysis of chromosome 4 of the plant Arabidopsis thaliana. Nature 402: 769-777.

Meinke, D.W., Cherry, J.M., Dean, C., Rounsley, S.D., and Koornneef M. 1998. Arabidopsis thaliana: A model plant for genome analysis. Science 282: 662, 679-682.

Mercier, R., Jolivet, S., Vezon, D., Huppe, E., Chelysheva, L., Giovanni, M., Nogue, F., Doutriaux, M.P., Horlow, C., Grelon, M., et al. 2005. Two meiotic crossover classes cohabit in Arabidopsis: One is dependent on MER3, whereas the other one is not. Curr. Biol. 15: 692-701.

Meunier, J. and Duret, L. 2004. Recombination drives the evolution of GC-content in the human genome. Mol. Biol. Evol. 21: 984-990.

Montoya-Burgos, J.I., Boursot, P., and Galtier, N. 2003. Recombination explains isochores in mammalian genomes. Trends Genet. 19: $128-130$.

Nachman, M.W. 2002. Variation in recombination rate across the genome: Evidence and implications. Curr. Opin. Genet. Dev. 12: $657-663$.
Okagaki, R.J. and Weil, C.F. 1997. Analysis of recombination sites within the maize waxy locus. Genetics 147: 815-821.

Petes, T.D. and Merker, J.D. 2002. Context dependence of meiotic recombination hotspots in yeast: The relationship between recombination activity of a reporter construct and base composition. Genetics 162: 2049-2052.

Quesneville, H., Bergman, C.M., Andrieu, O., Autard, D., Nouaud, D., Ashburner, M., and Anxolabehere, D. 2005. Combined evidence annotation of transposable elements in genome sequences. PLoS Comput. Biol. 1: e22.

Rafalski, A. and Morgante, M. 2004. Corn and humans: Recombination and linkage disequilibrium in two genomes of similar size. Trends Genet. 20: 103-111.

Sauer, S., Lechner, D., Berlin, K., Lehrach, H., Escary, J.L., Fox, N., Gut, I.G. 2000. A novel procedure for efficient genotyping of single nucleotide polymorphisms. Nucleic Acids Res. 28: E13.

Sanchez-Moran, E., Armstrong, S.J., Santos, J.L., Franklin, F.C., and Jones, G.H. 2002. Variation in chiasma frequency among eight accessions of Arabidopsis thaliana. Genetics 162: 1415-1422.

Schmidt, R., West, J., Love, K., Lenehan, Z., Lister, C., Thompson, H., Bouchez, D., and Dean, C. 1995. Physical map and organization of Arabidopsis thaliana chromosome 4. Science 270: 480-483.

Schnable, P.S., Hsia, A.P., and Nikolau, B.J. 1998. Genetic recombination in plants. Curr. Opin. Plant Biol. 1: 123-129.

Stahl, F.W., Foss, H.M., Young, L.S., Borts, R.H., Abdullah, M.F., and Copenhaver, G.P. 2004. Does crossover interference count in Saccharomyces cerevisiae? Genetics 168: $35-48$.

Takano-Shimizu, T. 2001. Local changes in GC/AT substitution biases and in crossover frequencies on Drosophila chromosomes. Mol. Biol. Evol. 18: 606-619.

Wright, S.I., Agrawal, N., and Bureau, T.E. 2003. Effects of recombination rate and gene density on transposable element distributions in Arabidopsis thaliana. Genome Res. 13: 1897-1903.

Yao, H., Zhou, Q., Li, J., Smith, H., Yandeau, M., Nikolau, B.J., and Schnable, P.S. 2002. Molecular characterization of meiotic recombination across the 140-kb multigenic a1-sh2 interval of maize. Proc. Natl. Acad. Sci. 16: 16.

Yu, A., Zhao, C.F., Fan, Y., Jang, W.H., Mungall, A.J., Deloukas, P., Olsen, A., Doggett, N.A., Ghebranious, N., Broman, K.W., et al. 2001. Comparison of human genetic and sequence-based physical maps. Nature 409: 951-953.

Zickler, D. and Kleckner, N. 1999. Meiotic chromosomes: Integrating structure and function. Annu. Rev. Genet. 33: 603-754.

\section{Web site references}

http://www.arabidopsis.org/; The Arabidopsis information resource. http://ftp.tigr.org/pub/data/a_thaliana/ath1/; Arabidopsis sequence database.

http://cran.r-project.org; R software.

Received June 20, 2005; accepted in revised form September 28, 2005.

114 Genome Research

www.genome.org 


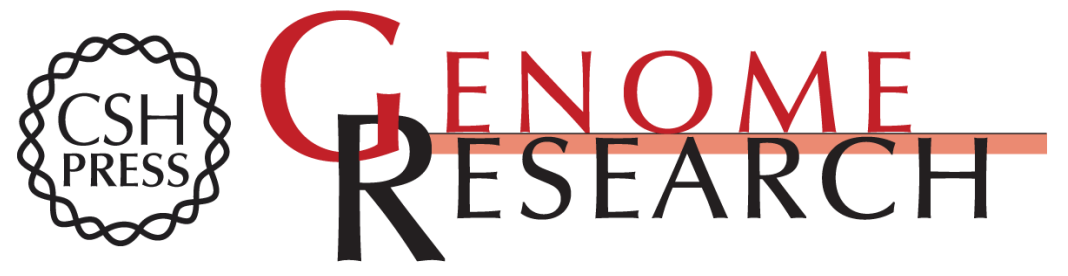

\title{
Variation in crossing-over rates across chromosome 4 of Arabidopsis thaliana reveals the presence of meiotic recombination "hot spots"
}

Jan Drouaud, Christine Camilleri, Pierre-Yves Bourguignon, et al.

Genome Res. 2006 16: 106-114

Access the most recent version at doi:10.1101/gr.4319006

Supplemental http://genome.cshlp.org/content/suppl/2005/12/13/gr.4319006.DC1
Material

References This article cites 63 articles, 22 of which can be accessed free at:

http://genome.cshlp.org/content/16/1/106.full.html\#ref-list-1

\author{
License
}

Email Alerting Receive free email alerts when new articles cite this article - sign up in the box at the Service top right corner of the article or click here.

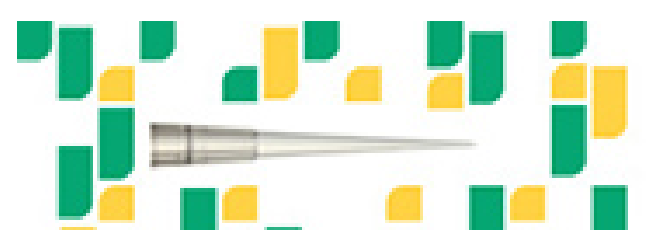

To subscribe to Genome Research go to: https://genome.cshlp.org/subscriptions 